BULLETIN OF THE

AMERICAN MATHEMATICAL SOCIETY

Volume 77, Number 2, March 1971

\title{
FINITELY GENERATED NILPOTENT GROUPS WITH ISOMORPHIC FINITE QUOTIENTS
}

\author{
BY P. F. PICKEL ${ }^{1}$
}

Communicated by Armand Borel, August 10, 1970

Let $G$ be a finitely generated nilpotent group and let $\mathfrak{F}(G)$ denote the set of isomorphism classes of finite homomorphic images of $G$. If $H$ is another finitely generated nilpotent group, we will say that $G$ and $H$ have isomorphic finite quotients if $\mathfrak{F}(G)=\mathfrak{F}(H)$. The finite quotients of a finitely generated nilpotent group provide much information about the structure of the group [3], [5], although they do not determine the group up to isomorphism [8, and G. Higman, unpublished]. The following result shows, however, that a finitely generated nilpotent group is determined to a large extent by its finite quotients.

TheOREM. Let $G$ be a finitely generated nilpotent group. Then the finitely generated nilpotent groups $H$, for which $\mathfrak{F}(G)=\mathfrak{F}(H)$, lie in only finitely many isomorphism classes.

This theorem, which is a much stronger version of an unpublished result of A. Borel, is proved by using the Lie algebras of the respective nilpotent groups [6], [7] to apply some finiteness results for arithmetic subgroups of algebraic groups [4], a technique introduced by Auslander and Baumslag [1], [2].

OUtLINe of the Proof. We first give some necessary notation and state a few fundamental lemmas. If $G$ is a finitely generated nilpotent group, we can define a $p$-adic topology on $G$ for which a neighborhood basis of the identity is given by the groups $G^{p^{i}}=\operatorname{gp}\left\{x^{p^{i}} \mid x \in G\right\}$. The completion of $G$ in this topology will be denoted $Z_{p} G$. The connection between these completions and finite quotients is given by the following lemma of Borel:

LEMMA 1. If $G$ and $H$ are finitely generated nilpotent groups, then $\mathfrak{F}(G)=\mathfrak{F}(H)$ iff $Z_{p} G$ and $Z_{p} H$ are isomorphic for each prime $p$.

If $N$ is a subgroup of $G$, then $Z_{p} N$ may be considered to be a sub-

AMS 1969 subject classifications. Primary 2040, 2027.

Key words and phrases. Isomorphic finite quotients, arithmetic groups, algebraic groups, Lie algebras.

1 A generalization of part of the results of the author's doctoral dissertation written under the guidance of G. Baumslag at Rice University, where the author was supported by a Rice Fellowship.

Copyright @ 1971, American Mathematical Society 
group of $Z_{p} G$ and if $N$ is normal in $G, Z_{p} N$ is normal in $Z_{p} G$ and $Z_{p}(G / N) \cong Z_{p} G / Z_{p} N$. Using this and Lemma 1 , it is easy to prove the following lemma which allows us to reduce to the torsion free case.

LemMa 2. If two finitely generated nilpotent groups $G$ and $H$ have isomorphic finite quotients, then their torsion subgroups $\tau G$ and $\tau H$ are isomorphic and the respective torsion free factor groups $G^{*}=G / \tau G$ and $H^{*}=H / \tau H$ have isomorphic finite quotients.

If $G$ is a torsion free finitely generated nilpotent group (an $N$-group for short), then $Z_{p} G$ is easily seen to be torsion free so we may form the Mal'cev completions $Q G$ of $G$ and $Q_{p} G$ of $Z_{p} G$ [11, p. 256]. $G$ and $H$ are commensurable $N$-groups if and only if $Q G$ and $Q H$ are isomorphic [7]. It is not too hard to check that if $\phi$ is an isomorphism of $G$ onto $H, \phi$ extends uniquely to isomorphisms $\phi_{p}: Z_{p} G \rightarrow Z_{p} H$ and $\bar{\phi}: Q G \rightarrow Q H$. Also isomorphisms $\phi_{p}: Z_{p} G \rightarrow Z_{p} H$ extend uniquely to isomorphisms $\phi_{p}: Q_{p} G \rightarrow Q_{p} H$ and an isomorphism $\psi: Q G \rightarrow Q H$ extends uniquely to isomorphisms $\psi_{p}: Q_{p} G \rightarrow Q_{p} H$. We then may show:

Lemma 3. If $G$ and $H$ are $N$-groups and $\psi: Q G \rightarrow Q H$ is an isomorphism, then for all but a finite number of primes $p$, the extension $\psi: Q_{p} G$ $\rightarrow Q_{p} H$ sends $Z_{p} G$ isomorphically onto $Z_{p} H$.

If $H$ is a subgroup of finite index in an $N$-group $G$, it is easy to see that $Z_{p} H$ is of finite index in $Z_{p} G$. This fact is used in a rather long argument following [9] to show:

Lemma 4. If $H$ is a subgroup of finite index in an $N$-group $G$, then there is a subgroup $K$ of $\operatorname{Aut}\left(Q_{p} G\right)$ of finite index in each of $\operatorname{stab}\left(Z_{p} G, \operatorname{Aut}\left(Q_{p} G\right)\right)$ and $\operatorname{stab}\left(Z_{p} H, \operatorname{Aut}\left(Q_{p} G\right)\right)$.

If $R$ is the rational group ring of an $N$-group $G$ and $B$ is its augmentation ideal, $B$ is residually nilpotent [6] so we may form the completion $R^{\wedge}$ of $R$ in a Hausdorff $B$-adic topology. If $x$ is an element of $B^{\wedge}$, the usual power series for $\exp (x)$ and $\log (1+x)$ converge in $R^{\wedge}$ giving inverse maps between the sets $B^{\wedge}$ and $1+B^{\wedge}$. Since $G$ is contained in $1+B^{\wedge}$, we may consider the $Q$-vector space $\Lambda$ spanned by $\log (G)$ in $B^{\wedge} . \Lambda$ is a finite dimensional nilpotent Lie subalgebra of the commutation Lie algebra on $R$ [6]. We call $\Lambda$ the Lie algebra of $G$. If $\log (G)$ is an additive lattice in $\Lambda$, we say $G$ is lattice nilpotent [12].

If $\Gamma$ is any nilpotent Lie algebra over a field $F$ of characteristic zero, we may define a multiplication $*$ on $\Gamma$ using the Baker-CampbellHausdorff formula so that the group $(\Gamma, *)$ is a nilpotent group admitting the action of $F$ (as a set) [6]. If $\Lambda$ is the Lie algebra of the $N$-group $G$, exp gives an isomorphism of the subgroup $(\log G, *)$ with 
$G$ and exp takes $(\Lambda, *)$ isomorphically onto a Mal'cev completion of $G$ contained in $R^{\wedge}$ [6], [2]. In general, if $\Lambda$ and $\Gamma$ are nilpotent Lie algebras over a field $F$ (char 0 ) and $\phi$ is a bijective map of $F$-sets, a tedious double induction shows that $\phi$ is a Lie algebra isomorphism iff $\phi$ is a group theoretic isomorphism of $(\Lambda, *)$ onto $(\Gamma, *)$. Using this we may prove the following crucial propositions.

Proposition 5 (BOREL). Suppose $G$ and $H$ are $N$-groups with Lie algebras $\Gamma$ and $\Lambda$ respectively. Then $Q G \cong Q H$ iff $\Gamma \cong \Lambda$ and $Q_{p} G \cong Q_{p} H$ iff $Q_{p} \otimes_{Q} \Gamma \cong Q_{p} \otimes_{Q} \Lambda$.

Proposition 6. If $G$ is a lattice nilpotent group, there is an algebraic matric group (5) $[4, p$. 10] and, for each prime $p$, isomorphisms $\delta_{p}: \operatorname{Aut}\left(Q_{p} G\right) \rightarrow \mathbb{S}_{Q_{p}}$ which take $\operatorname{stab}\left(Z_{p} G, \operatorname{Aut}\left(Q_{p} G\right)\right)$ to $\mathbb{S}_{Z_{p}}$, $\operatorname{stab}\left(Q G, \operatorname{Aut}\left(Q_{p} G\right)\right)$ to $\mathbb{S}_{Q}$ and $\operatorname{stab}\left(G\right.$, Aut $\left.Q_{p} G\right)$ to $\mathbb{H}_{z}$ in such a way that for $\phi$ in $\operatorname{Aut}(Q G), \delta_{p}(\phi)$ is independent of $p$ in $\mathfrak{B S}_{Q}$. $\left(\mathbb{S}_{Q}\right.$ is $\left.\operatorname{Aut}(\Lambda).\right)$

The following unpublished theorem of A. Borel, which was the major motivation for this work, may be proved using Lemma 1, Proposition 5, and Theorem 7.11 of [10].

TheOREM 7 (BOREL). Let $G$ be an $N$-group. Then the $N$-groups $H$ for which $\mathfrak{F}(G)=\mathfrak{F}(H)$ are contained in finitely many commensurability classes.

For any $N$-group $G$, we define $\Theta_{A}$ to be the subgroup of $\Pi_{p} \operatorname{Aut}\left(Q_{p} G\right)$ consisting of elements $\Pi\left(\alpha_{p}\right)$ for which $\alpha_{p} \in \operatorname{stab}\left(Z_{p} G, \operatorname{Aut}\left(Q_{p} G\right)\right)$ for all but a finite number of primes $p$. We define $\Theta_{A}^{\infty}$ to be the subgroup of $\oint_{A}$ consisting of elements $\Pi\left(\alpha_{p}\right)$ such that $\alpha_{p} \in \operatorname{stab}\left(Z_{p} G\right.$, Aut $\left.\left(Q_{p} G\right)\right)$ for all primes $p$. By Lemma 3 we may embed $\operatorname{Aut}(Q G)$ diagonally as a subgroup $G_{Q}$ of $G_{A}$.

If $H$ and $G$ are $N$-groups, we say $H$ is in the genus of $G$ if $\mathfrak{F}(G)$ $=\mathfrak{F}(H)$ and $H$ is commensurable with $G$. If so, we have isomorphisms $\phi_{p}: Z_{p} G \rightarrow Z_{p} H$ and $\psi: Q G \rightarrow Q H$ which extend to $\bar{\phi}_{p}, \psi_{p}: Q_{p} G \rightarrow Q_{p} H$. By Lemma $3, \Pi\left(\bar{\phi}_{p}^{-1} \circ \psi_{p}\right)$ is in $\oint_{A}$. Using this construction and following the idea of Proposition 2.3 of [4] we may show:

Proposition 8. The isomorphism classes in the genus of an $N$-group $G$ are in 1-1 correspondence with a subset of the set of double cosets $\mathcal{G}_{A}^{\infty} \backslash \mathcal{S}_{A} / \mathcal{S}_{\boldsymbol{Q}}$.

If $G$ is lattice nilpotent, Proposition 6 and Theorem 5.1 of [4] imply that the number of double cosets is finite. If not, $G$ is a subgroup of finite index in a lattice nilpotent group $H$ [12, Theorem 2]. We then have $\mathcal{S}_{A}=\mathfrak{K}_{A}, \mathcal{S}_{Q}=\mathfrak{K}_{Q}$ and by Lemmas 3 and $4, \mathcal{S}_{A}^{\infty}$ and $\mathfrak{K}_{A}^{\infty}$ are 
commensurable. Thus in this case the number of double cosets is again finite so we have:

THEOREM 9. There are only finitely many isomorphism classes of $N$ groups in the genus of an $N$-group $G$.

The main theorem then follows from Lemma 2, Theorem 7 and Theorem 9.

\section{REFERENCES}

1. L. Auslander and G. Baumslag, Automorphism groups of finitely generated nilpotent groups, Bull. Amer. Math. Soc. 73 (1967), 716-717. MR 36 \#259.

2. G. Baumslag, Notes on nilpotent groups, Conference Board Math. Sci., Regional Conference Ser. Math. No. 2, Amer. Math. Soc., Providence, R. I., 1971.

3. N. Blackburn, Conjugacy in nilpotent groups, Proc. Amer. Math. Soc. 16 (1965), 143-148. MR $30 \# 3140$.

4. A. Borel, Some finiteness properties of adele groups over number fields, Inst. Hautes Etudes Sci. Publ. Math. No. 16 (1963), 5-30. MR 34 \#2578.

5. K. A. Hirsch, On infinite soluble groups. II, Proc. London Math. Soc. (2) 44 (1938), 336-344.

6. S. A. Jennings, The group ring of a class of infinite nilpotent groups, Canad. J. Math. 7 (1955), 169-187. MR 16, 899.

7. A. I. Mal'cev, On a class of homogeneous spaces, Izv. Akad. Nauk SSSR Ser. Mat. 13 (1949), 9-32; English transl., Amer. Math. Soc. Transl. (1) 9 (1962), 276307. MR 10, 507.

8. V. N. Remeslemnikov, Conjugacy of subgroups in nilpotent groups, Algebra $\mathbf{i}$ Logika Sem. 6 (1967), no. 2, 61-76. (Russian) MR 36 \#1545.

9. G. Baumslag, Automorphism groups of nilpotent groups, Amer. J. Math. 91 (1969), 1003-1011.

10. A. Borel and J. P. Serre, Theorèmes de finitude en cohomologie galoisienne, Comment. Math. Helv. 39 (1964), 111-164. MR 31 \#5870.

11. A. G. Kuroš, The theory of groups, 2nd ed., GITTL, Moscow, 1953; English transl., Chelsea, New York, 1955. MR 15, 501; MR 17, 124.

12. C. C. Moore, Decomposition of unitary representations defined by discrete subgroups of nilpotent Lie groups, Ann. of Math. (2) 82 (1965), 146-182. MR 31 \#5928.

Rice University, Houston, Texas 77001 